\title{
DEVELOPMENT OF SEMICONDUCTOR DETECTORS FOR VERY HARSH RADIATION ENVIRONMENTS IN HIGH ENERGY PHYSICS APPLICATIONS
}

G. Casse

O. Lodge Laboratory - University of Liverpool, on behalf of the RD50 collaboration. Full author list

at http://www.cern.ch/rd50

The Large Hadron Collider (LHC) at CERN has been designed to achieve the unprecedented luminosity of $10^{34} \mathrm{~cm}^{-2} \mathrm{~s}^{-1}$. As a consequence, the silicon detectors close to the interaction region will receive severe doses of hadron irradiation. The present sensors are designed to survive fast hadron fluences of about $10^{15} \mathrm{~cm}^{-2}$. Due to the anticipated radiation levels, the fluence expected at the innermost tracker detectors can already exceed this value before the end of the lifetime of the experiment, so that some experiments foresee to change these detectors after a few years of operation (e.g. LHCb VELO). Moreover, the option of increasing the luminosity of LHC to $10^{35}$ $\mathrm{cm}^{-2} \mathrm{~s}^{-1}$ has been envisaged to extend the physics reach of the machine. An efficient tracking down to a few centimetres from the interaction point will be required to exploit the physics potential of the upgraded LHC. Under these conditions, the inner tracker detectors will need to survive fast hadron fluences above $10^{16} \mathrm{~cm}^{-2}$. The CERN-RD50 project "Development of Radiation Hard Semiconductor Devices for Very High Luminosity Collider" has been set-up to explore detector technologies that will allow to operate devices up to, or beyond, this limit.

The strategies followed by RD50 to enhance the radiation tolerance include the development of new or defect engineered detector materials ( $\mathrm{SiC}, \mathrm{GaN}, \mathrm{CZ}$ and EPI silicon, oxygen enriched silicon), the evaluation of new detector designs (3D, Semi-3D detectors), the improvement of present detector designs and, on the fundamental semiconductor physics aspect, the understanding of the microscopic defects causing the degradation of the irradiated detectors.

The latest advancements within the RD50 collaboration will be reviewed and discussed in this work.

\section{Introduction to the CERN RD50 collaboration}

The future vertex detectors for very high luminosity colliders (as the possible upgrade of the LHC to a luminosity of $10^{35} \mathrm{~cm}^{-2} \mathrm{~s}^{-1}$ ) are expected to operate in an environment where the total fluences of fast hadrons will be above $10^{16} \mathrm{~cm}^{-2}$. The present vertex detectors rely on highly segmented silicon sensors to achieve the accuracy required by the physics performances. Semiconductor detectors seem the best option for vertex sensors also in the next generation of colliders, provided that their radiation hardness will be improved. The RD50 collaboration is dedicated to the development of such a radiation hard semiconductor detectors. The collaboration has been approved in June 2002 by the CERN research board and it counts a total of 280 members with 55 participant institutes from 47 countries in Europe, 7 from North America (Canada, USA) and one from middle east (Israel).

Two main research lines have been identified to achieve these goals, Material Engineering and Device Engineering, and each of them has been divided in three projects, as summarised in Table 1. The aim is to produce the most 
intrinsically radiation hard semiconductor material (Material Engineering) in term of degradation of the electrical properties after irradiation and to identify the best geometry (size and position) of the segmented electrodes in order to optimise the radiation resistance of any particular semiconductor material (Device Engineering). The activity of each research line of RD50 is here presented.

\begin{tabular}{|c|c|c|c|c|c|}
\hline \multicolumn{3}{|c|}{ Material engineering } & \multicolumn{3}{c|}{ Device engineering } \\
\hline $\begin{array}{c}\text { Defect } \\
\text { characterisation }\end{array}$ & $\begin{array}{c}\text { Defect } \\
\text { engineering }\end{array}$ & $\begin{array}{c}\text { New } \\
\text { materials }\end{array}$ & $\begin{array}{c}\text { Pad detector } \\
\text { characterisation }\end{array}$ & $\begin{array}{c}\text { Full detector } \\
\text { systems }\end{array}$ & $\begin{array}{c}\text { New } \\
\text { structures }\end{array}$ \\
\hline
\end{tabular}

Table 1. Main research lines and their relative research projects in RD50.

\section{Defect characterisation}

The degradation of silicon detectors with irradiation is caused by lattice defects like creation of vacancies (point-like defect) or of damaged regions (clusters) that interact with other defects or impurities to form electrically active stable centres. Only point-like defects are created under 60Co- $\gamma$ irradiation, while both type of defects are created with hadron irradiation, and there ratio depends on the particle type and energy [1]. The knowledge of the primary defect creations and of their evolution with time to form stable complexes with other defects and impurities would inform the deliberate introduction of particular impurities in the crystal to reduce the degradation of the electrical properties of the devices. A crucial aspect for the understanding of the defect kinetics at a microscopic level is the correct identification of the crystal defects in term of their electrical activity.

The main tools available for studying the defect kinetics are the Deep Level Transient Spectroscopy (DLTS) and the Thermally Stimulated Current (TSC). Important advancements in understanding the radiation damage in the case of point defects have been performed within the RD50 collaboration. The point defects of crucial importance for the radiation hardness of silicon detectors have been studied with DLTS and TSC after irradiation with 60Co- $\gamma$. This investigation has shown for the first time evidence on the formation of the defects responsible for the change in the effective doping concentration $\left(N_{\text {eff }}\right.$, and therefore of the full depletion voltage $V_{f d}$ ), and in the free carrier generation (and therefore of the reverse current, $I_{R}$ ). Three defects have been correlated with these changes, namely two midgap acceptor levels $(I$ and $\Gamma$ ) and a bi-stable donor $(B D) . I$ and $\Gamma$ are generated in higher concentrations in standard FZ 
silicon than in oxygen enriched silicon, while the $B D$ is found in oxygen enriched and epitaxial silicon (see Fig. 1) ([2-4]).

The $\Gamma$ defect has an acceptor level at $\mathrm{E}_{\mathrm{v}}+0.68 \mathrm{eV}$, linear dose dependence and can explain about $10 \%$ of the damage. The $I$ defect is of amphoteric nature having both an acceptor state at $\mathrm{E}_{\mathrm{c}}-0.54 \mathrm{eV}$ and a donor state at $\mathrm{E}_{\mathrm{v}}+0.23 \mathrm{eV}$, has a quadratic dose dependence (Fig.1b) and it is proven to be the main cause for the observed inversion of the space charge sign in the depletion region of standard FZ diodes (it accounts for more than $85 \%$ of the damage caused by gammas).
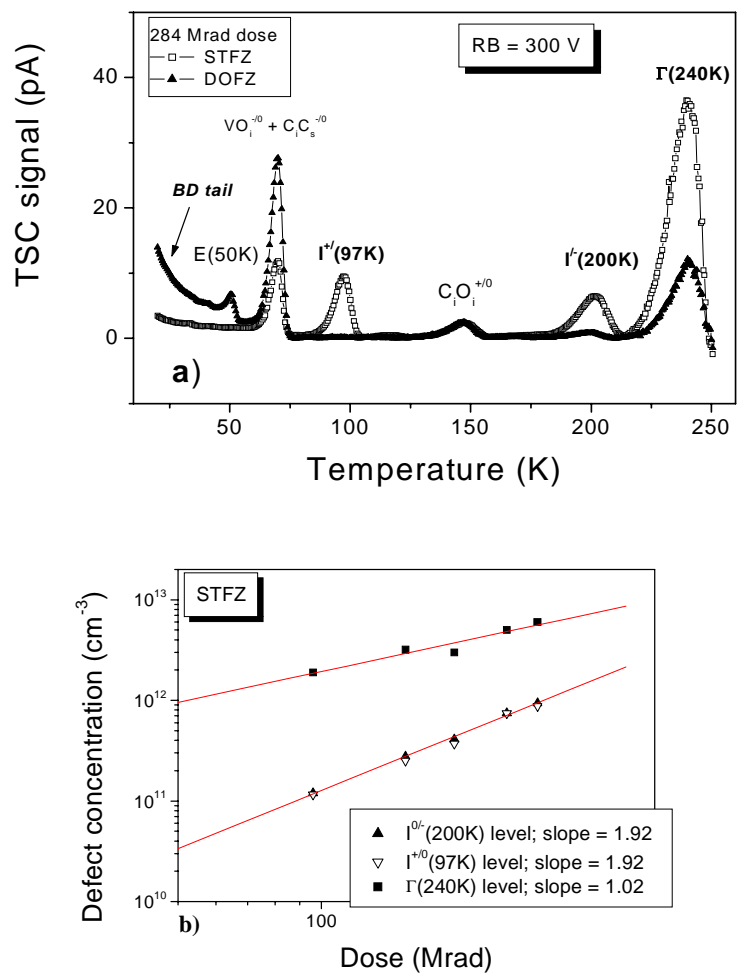

Figure 1: a) TSC spectra for standard FZ (STFZ) and oxygen enriched (DOFZ) silicon [3]; b) dose dependence of $I$ and $\Gamma$ defects [4].

Concerning its chemical composition, the $I$ level is a good candidate to be associated with the divacancy-oxygen $\left(\mathrm{V}_{2} \mathrm{O}\right)$ centre, based on experimental data and kinetic modelling results, but further investigation is needed to support this hypothesis. 
The formation of the third identified centre, the bistable donor $B D$, is largely enhanced in epitaxial diodes. These diodes show superior radiation tolerance compared to all types of float zone devices when irradiated with protons and they don't show the conductivity type inversion at any fluence (see below, $\S$ 3.2). This can be attributed to the formation of the BD centre, present in the epitaxial substrate, as shown in Fig. 2 in the $80-100 \mathrm{~K}$ range, and to the absence of the $I$-level, in contrast to the float zone substrate.

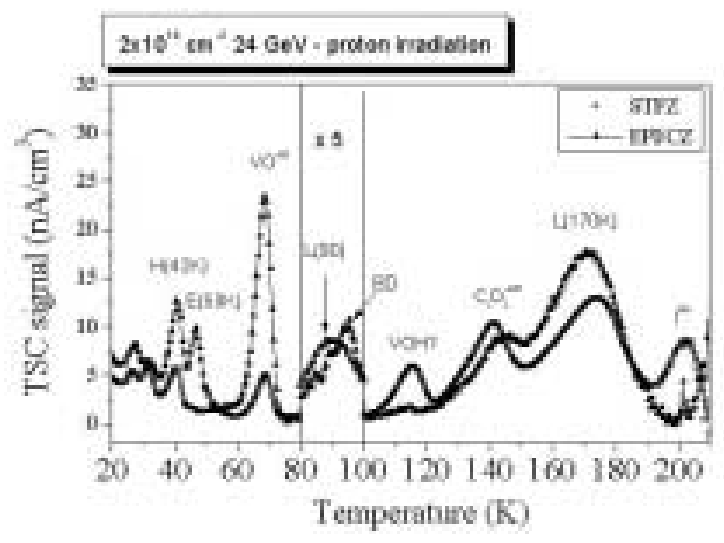

Figure 2 TSC spectra measured after proton irradiation [5].

A tentative explanation for the donor generation in epitaxial diodes is based on the fact that oxygen dimers $\left(O_{2 i}\right)$ present in the $\mathrm{Cz}$-substrate will migrate into the epitaxial layer during the growth process, such that the ratio of dimers to monoatomic interstitial oxygen $\left(O_{i}\right)$ is much higher in epitaxial silicon than in standard or oxygenated FZ silicon. Dimers are known to be precursors for irradiation induced thermal donor generation thus leading to an increase of the net positive space charge, more than balancing the negative space charge generation seen in FZ devices due to generation of deep acceptors. Further studies, using oxygen dimer enriched silicon could help on the identification of these donor levels.

\section{Defect engineering}

\subsection{High resistivity Czochralski silicon}

The CERN RD48 (ROSE) [6] collaboration showed that oxygen enriched silicon substrates exhibit a considerable improvement in the change of the effective space charge concentration after charged hadron and gamma irradiation while no or little benefit is found after exposure to neutrons [7]. The model used to explain the behaviour of the oxygen enriched silicon identifies the $\mathrm{V}_{2} \mathrm{O}$ defect as 
the main responsible for the radiation induced negative space charge. The formation of $\mathrm{V}_{2} \mathrm{O}$ is suppressed in oxygen rich material [8], through the reaction of interstitial oxygen with vacancies. The reaction that leads to the formation of $\mathrm{V}_{2} \mathrm{O}$ is: $\mathrm{V}+\mathrm{O}_{\mathrm{i}} \rightarrow \mathrm{VO}, \mathrm{VO}+\mathrm{V} \rightarrow \mathrm{V}_{2} \mathrm{O}$.

Increasing the concentration of interstitial oxygen enhances the ratio of the first of the two reactions, reducing the formation of $\mathrm{V}_{2} \mathrm{O}$. The model predictions support also the experimental observation that the effect of oxygen is correlated with the radiation-induced formation of point defects. The neutron damage produces more cluster defects and no improvement due to the oxygen enrichment is expected, while maximum suppression of the radiation damage is anticipated when only point defects are created, as for ${ }^{60} \mathrm{Co}-\gamma$ irradiation $[9,10]$. The results from the RD48 collaboration where mainly obtained with oxygen enriched $\mathrm{FZ}$ silicon wafers via high temperature diffusion from a $\mathrm{SiO}_{2}$ layer grown on the wafer surface (DOFZ)[11,12]. The maximum concentration achieved with this technique was $\sim 2-4 \times 1010^{17} \mathrm{~cm}^{-3}$. Higher oxygen concentration $\left([\mathrm{O}] \approx 4-2010^{17} \mathrm{~cm}^{-3}\right)$ is found in silicon crystals grown by the Czochralski (Cz) method. High resistivity $\mathrm{CZ}$ suitable for detector application is now available after recent developments in the crystal growth technique (see e.g. [13]). Possible improvement in the radiation hardness beyond the level observed so far in DOFZ silicon can be expected, due to the higher $O_{i}$ content and possibly to the presence of oxygen dimers $\left(O_{2 i}\right)$ [14]. Two different $\mathrm{Cz}$ materials have been investigated in the framework of RD50: Magnetic Czochralski (MCz) from Okmetic Ltd Finland and standard $\mathrm{Cz}(\mathrm{SCz})$ material from SumitomoSitix (Japan). The first material has a resistivity of $\approx 900 \Omega \mathrm{cm}$ and an oxygen concentration of $3.510^{17} \mathrm{~cm}^{-3}$ and the second has a resistivity of $\approx 1200 \Omega \mathrm{cm}$ and an oxygen concentration of $810^{17} \mathrm{~cm}^{-3}$.

The MCz silicon was processed by the Helsinki Institute of Physics (HIP) to manufacture pad diodes and microstrip detectors, while the $\mathrm{SCz}$ was processed by CiS, Germany, in collaboration with the Hamburg University.

Both materials have been characterized by DLTS after processing, exhibiting some difference between them. For the $\mathrm{MCz}$ material two deep electron trap levels were detected and associated with thermally induced defects [15]. For the $\mathrm{SCz}$ devices a shallow level at $\mathrm{E}_{\mathrm{C}}-0.12 \mathrm{eV}$ was detected while no deep levels were found. The shallow level is associated with thermal double donors (TDD). For a limited set of devices thermal donors were intentionally activated by thermal treatment at $400^{\circ} \mathrm{C}$ for $40 \mathrm{~min}$. Due to this treatment the effective doping concentration changed from $3.310^{12} \mathrm{~cm}^{-3}$ to $7.110^{12} \mathrm{~cm}^{-3}[16]$.

The radiation hardness of both materials has been investigated by several irradiation campaigns at different facilities and the results are reported later (see $\S 3.1$.

\subsection{Thin epitaxial silicon layers}

A different approach for increasing the radiation tolerance of silicon detectors is the use of thin epitaxial silicon layers. Single crystal epitaxial layers of different 
resistivity and thickness can be grown on different substrates (Cz, FZ). Low resistivity $(50 \Omega \mathrm{cm}) 50 \mu \mathrm{m}$ thick epitaxial layers were grown by ITME (Poland) on low resistivity $(0.015 \Omega \mathrm{cm}) \mathrm{Cz}$ substrates [17].

Due to the high oxygen content in the $\mathrm{Cz}$ substrate and the high temperature during the epitaxial growth process, oxygen atoms diffuse into the epi-layer with an average concentration of about $9.10^{16} \mathrm{~cm}^{-3}$. Several irradiation experiments with protons of different energy, reactor neutrons, Li-ions, high energy electrons and ${ }^{60} \mathrm{Co}$ gamma rays have been performed, exhibiting the excellent radiation hardness properties of diodes made with epitaxial material [2]. Results after proton and neutron irradiations are presented in $\$ 3.2$.

\subsection{Oxygen dimers in silicon}

As already mentioned, $O_{2 i}$ enriched silicon could be a route towards radiation hardening of silicon detectors, though very little is known at the moment about the possible advantages of this material. The idea is to produce a silicon material that contains oxygen dimers $\left(O_{2 i}\right)$ instead of mono atomic oxygen $\left(O_{i}\right)$. This new material is expected to have different defect kinetics during irradiation. For example it can influence the reaction of vacancies and reduce the formation of the $\mathrm{V}_{2} \mathrm{O}$ defect while alternatively leading to the formation of the $\mathrm{VO}_{2}$ defect, known to be neutral and possibly less damageable to detectors. Furthermore, $O_{2 i}$ can act as a sink for migrating interstitials I by forming the $I_{2 i}$ defect, detected in $\mathrm{Cz}$ [18] as well as in DOFZ Si [4]. It is now generally accepted that $O_{2 i}$ are precursors for the formation of earlier stage thermal donors [19] and such irradiation induced bistable donors were already detected in DOFZ and epi-Si (see $\S 1$ ).

RD50 has launched an experiment to produce dimers in different kind of silicon material and test the radiation hardness of dimer-rich materials using $24 \mathrm{GeV} / \mathrm{c}$ protons at the CERN PS [2].

$O_{2 i}$ can be produced in the silicon crystal by carefully playing with the existing oxygen impurities and other defects present in the original material. This can be achieved by creating point defects in the crystal (with e.g. low energy electrons), and by forming $O_{2 i}$ through the following reactions with vacancies and interstitials: $V O_{i}+O_{i} \rightarrow V O_{2 i}, I+V O_{2 i} \rightarrow O_{2 i}$.

It is possible to convert $\mathrm{O}_{\mathrm{i}}$ into $\mathrm{O}_{2 \mathrm{i}}$ [20] in silicon by irradiating the material with electrons of $2.5 \mathrm{MeV}$ at $350{ }^{\circ} \mathrm{C}$ up to a fluence of $1.10^{18} \mathrm{e} / \mathrm{cm}^{2}$. The RD50 dimerisation process was performed using the $6 \mathrm{MeV}$ electrons beam at the KTH (Stockholm) facility on three types of diodes: standard float zone (initial depletion voltage around $60 \mathrm{~V}$ ), oxygenated float zone (initial depletion voltage around $50 \mathrm{~V}$ ) and $\mathrm{Cz}$ material (initial depletion voltage around $200 \mathrm{~V}$ ).

After dimerisation the diodes where measured in term of Capacitance-Voltage $(\mathrm{C}-\mathrm{V})$ and Current-Voltage (I-V) characteristics. The dimerised diodes could not be fully depleted. $N_{\text {eff }}$ could be evaluated from the slope of $1 / \mathrm{C}^{2}$ vs voltage and used to compute $V_{f d}$. To assess the reliability of this method, the same technique was applied to the control samples and the inferred $V_{f d}$ was compared to the 
measured one. All diodes (control and dimerised samples) where than irradiated to different $24 \mathrm{GeV} / \mathrm{c}$ proton fluences up to $1.110^{14} \mathrm{p} / \mathrm{cm}^{2}$. The inferred values of $V_{f d}$ as a function of fluence were as expected for the control samples, while very different behaviours, depending on the oxygen content of the material were found in the dimer-rich samples: for the standard float zone diode the inferred $\mathrm{V}_{\mathrm{fd}}$ was in the order of $550 \mathrm{~V}$ and significantly decreased with fluence while for the oxygenated float zone and the $\mathrm{Cz}$ diodes, the inferred $\mathrm{V}_{\mathrm{fd}}$ is in the order of $100,000 \mathrm{~V}$, constant as a function of fluence. These preliminary results suggest that the dimerisation process does not lead to material suitable for particle detector. Further analyses are necessary to understand the new defect kinetics. An hypothesis is that the thermal donors (possibly chains of oxygen atoms) that are created alongside $O_{2 i}$ overwhelm the beneficial effects provided by the oxygen dimers.

\section{Pad Detector Characterization (PDC)}

Pad diodes are the simplest structure to study and compare the degradation of the properties of various silicon materials after irradiation. They are used to measure the changes of the full depletion voltage $\left(\mathrm{V}_{\mathrm{fd}}\right)$ and the reverse current with fluence and time, and the degradation of the charge collection properties with fluence. They are also used for the study of the microscopic behaviors, via TSC and DLTS. Here the recent results obtained with new materials studied within RD50 and already mentioned earlier are presented.

\subsection{Cz materials}

As described before, two different types of high resistivity $\mathrm{Cz}(\mathrm{SCz}$ and $\mathrm{MCz}$ ) have been used to produce detectors. The $\mathrm{Cz}$ devices have exhibited interesting radiation hardness properties. Extensive studies have been done with different particles and energies.

The results of all these experiments can be summarised as it follows:

MCz devices [21,22]:

- For low energy protons $(10 \mathrm{MeV}, 20 \mathrm{MeV})$, type inversion is observed between $110^{14} \mathrm{~cm}^{-2}$ and $210^{14} \mathrm{~cm}^{-2} 1 \mathrm{MeV}$ neutron equivalent fluence.

- The reverse current increase seems to be smaller compared to standard FZ and DOFZ devices.

- After neutron irradiation the type inversion takes place between $310^{13} \mathrm{n} / \mathrm{cm}^{2}$ and $810^{13} \mathrm{n} / \mathrm{cm}^{2}$.

- After ${ }^{60} \mathrm{Co}$ gamma irradiation up to $1.2 \mathrm{Grad}$ there is no type inversion and $V_{f d}$ increases slowly with dose due to a build-up of positive space charge.

$S C z$ devices [23]: 
- There is no type inversion after irradiation with $190 \mathrm{MeV}$ pions at PSI and $24 \mathrm{GeV} / \mathrm{c}$ protons at CERN up to $110^{15}$ particles $/ \mathrm{cm}^{2}$. The change of $V_{f d}$ as function of fluence is considerably smaller than that of standard FZ or DOFZ silicon devices.

- No difference in the leakage current increase with fluence could be observed compared to standard FZ and DOFZ material.

- No difference in trapping time constants were observed by comparison to the standard FZ and DOFZ detectors. The degradation of CCE is about $15 \%$ after 6. $10^{14}$ pions $/ \mathrm{cm}^{2}$, as measured with a ${ }^{244} \mathrm{Cm} \alpha$-source.

A more detail compilation of the results obtained with the $\mathrm{Cz}$ devices can be found in [2] and references therein.

\subsection{Epitaxial diodes}

The epi-diodes show superior radiation tolerance compared to those fabricated with all other silicon materials. Figure 3 shows a comparison between epi-diodes and diodes fabricated with STFZ and DOFZ materials. In the full range of measured fluences (up to $\Phi_{\mathrm{eq}}=8.10^{14} \mathrm{~cm}^{-2}$ ) the epi-diodes do not exhibit type inversion and show a reduced variation of the full depletion voltage [16].

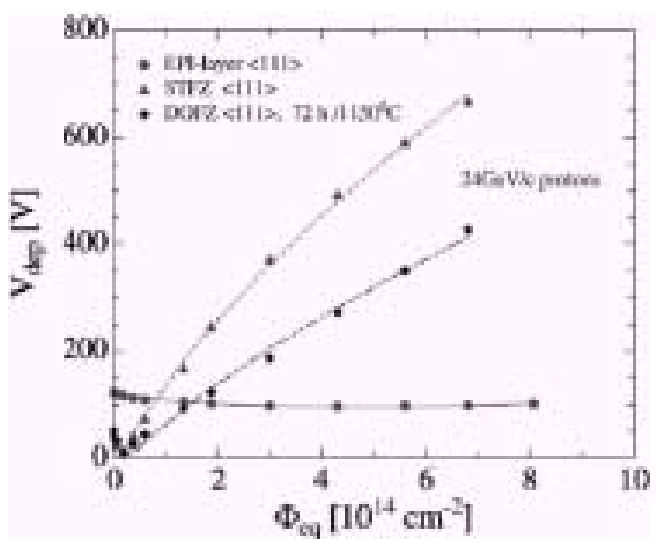

Figure 3. Comparison of epi-diodes and other materials as a function of $24 \mathrm{GeV} / \mathrm{c}$ fluence (CERN scenario) [16].

Annealing studies have also been performed and the results are shown in Fig. 4. The annealing curve shows the two components: fast component with decrease of $N_{\text {eff }}$ and slow component with increase of $N_{\text {eff }}$ up to a maximum of the reverse annealing. The annealing temperature of $80^{\circ} \mathrm{C}$ is used to speed up the process. It is possible to project the annealing curve at $80^{\circ} \mathrm{C}$ on the time scale at room temperature, as shown in the upper scale of Fig. 4. According to this prediction, 
the change of the depletion voltage with time at room temperature takes place slowly enough that the detectors made with epitaxial material may not need any cooling for LHC operation up to 10 years. This could be a tremendous advantage in term of maintenance and cost.

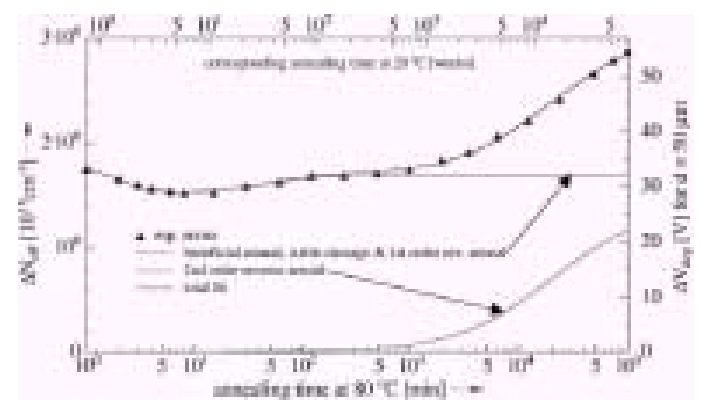

Figure 4. Annealing curve $\left(\Delta \mathrm{N}_{\text {eff }}\right)$ at $80 \mathrm{C}$ for epi-diodes after $24 \mathrm{GeV} / \mathrm{c}$ protons. The upper scale is the corresponding annealing time at 20C [16].

The absolute charge collection efficiency of epitaxial diodes as a function of fluence has been measured using alpha-particles $\left({ }^{244} \mathrm{Cm}\right)$ and a wide bandwidth $(<1 \mathrm{~ns}$ rising time) current amplifier. Regardless of the proton energy the studies show that for the epi-diodes the charge collection efficiency (CCE) drops to about $90 \%$ at $8.10^{14} / \mathrm{cm}^{2}$ while it drops to $70 \%$ for all other diodes at the same fluence. The CCE studies for epi-diodes are extended to $\Phi_{\text {eq }} \approx 10^{16} / \mathrm{cm}^{2}$. Even at this fluence (in the order of what expected at SuperLHC) the epi diodes are able to collect about $75 \%$ of the charge after proton irradiation and a slightly lower value after neutron irradiation.

\section{New structures}

In a standard detector geometry, electrodes are placed on opposite sides of the semiconductor, in a parallel plate capacitor geometry. The charge generated by ionising radiation drifts in the normal direction to the plane of the semiconductor wafer to the oppositely biased electrodes. The charge generated by the impinging radiation is proportional to the sensitive volume of the detector, therefore to the detector thickness when the device is fully depleted. When the detector suffers high radiation damage, the full depletion cannot be achieved anymore and the charge trapping reduces the amount of the collected charge drifting for longer distances.

A novel type of device (3D silicon detectors) was introduced [24] to reduce the electrode distance, therefore increasing the amount of charge collected after heavy irradiation, while keeping the same detector thickness. In $3 D$ devices, the 
electrodes are placed in holes drilled perpendicularly to the wafer surface throughout the detector bulk. The generated charge drifts in the plane of the wafer and the electrode separation is independent of the material thickness. Thus, the 3D geometry may be designed to both maximize signal response to incident radiation and minimize sensitivity to defects arising from radiation damage.

Three different routes to fabricating the 3D geometries have been developed. Depending on particular material system and requirements, one or other of these methods is to be preferred. The electrodes may be made using plasma etching (micro-machining), femtosecond laser drilling technique, or photoelectrochemical (PEC) etching.

The plasma etching technique is the most compatible with micro-fabrication processes. However, for 3D detector manufacture it works only for silicon and is limited to an aspect (depth-to-width) ratio for the holes currently of about 20:1. PEC will in principle produce holes of arbitrary aspect ratio, but the geometries usable are more limited due to the nature of the process. The laser drilling technique is the most cumbersome, as each electrode is made in sequence. In silicon, plasma etching and PEC produce electrodes in parallel, so both those methods are best suited for volume production of 3D detectors. Plasma etching and laser machining are independent of crystal orientation. PEC only works for specific crystal orientations which is easy commercially available from. The electrodes are than formed in these vias and connected to the read out electronics with deposited metal lines and contacts.

3D detectors have been produced at Glasgow by plasma etching with $200 \mu \mathrm{m}$ thick silicon and $85 \mu \mathrm{m}$ pore spacing and characterised with I-V and $\mathrm{C}-\mathrm{V}$ measurements. Several silicon 3D detectors were irradiated at the $300 \mathrm{MeV} / \mathrm{c}$ pion beam at the Paul Scherrer Institute (PSI), Villigen-CH, up to $10^{12}, 10^{13}$, and $5.10^{14} \mathrm{~cm}^{-2}$. The measurements show an improvement in both the leakage current and full depletion voltage with increased fluence, although the exact reason for this is not understood. The $\mathrm{C}-\mathrm{V}$ measurements show that full depletion is reached at progressively smaller voltages with higher fluence, going from the pre-irradiation value of $30 \mathrm{~V}$ to $20 \mathrm{~V}$ after the higher fluence. Pulse height spectra of these devices were measured before and after irradiation using $5.48 \mathrm{MeV} \alpha$ particles from a ${ }^{241} \mathrm{Am}$ source. The charge collection efficiency before irradiation was $\sim 60 \%$ (as measured with a calibrated system) and dropped to to $\sim 45 \%$ after irradiation. This results is slightly worse than what obtained with standard silicon diodes, probably due to non-optimised fabrication procedures. 
Besides the 3-D detectors, other new geometries are investigated within RD50, namely thin detectors [25] and semi-3D devices [26]. The status of these activities can be found in [2].

\section{Full detector systems}

The FDS research line of RD50 aims to test the properties of segmented detectors after heavy irradiation to allow to choose the optimal geometry (diode structure, detector thickness, cell size...) for the targeted radiation fluence and to validate the use of non-standard silicon substrates for the production of large area segmented devices. The crucial parameter that defines the ability of the detector to provide the tracking information is the signal over noise ratio. The noise is dependent on the particular set-up used to read-out the detectors, therefore the parameter chosen to evaluate the properties of the detectors after irradiation is the charge collection efficiency as a function of the applied bias voltage $(\mathrm{CCE}(\mathrm{V}))$.

The $\operatorname{CCE}(\mathrm{V})$ of irradiated silicon detectors depends on the trap density and on the collection time $\left(t_{c}\right)$, therefore on the drift velocity of the charge carriers. The amount of the trapped charge is inversely proportional to $t_{\mathrm{c}}$. The charge carrier velocity $v$ depends on the local electric field, $\mathrm{E}(\mathrm{x})$, like $\mathrm{v}=\mu_{\mathrm{e}, \mathrm{h}} \mathrm{E}(\mathrm{x})$ until saturation velocity. As a consequence a lower trapping is expected for carriers moving in high electric field (smaller $t_{c}$ ). Silicon detectors are usually manufactured by implanting high doped p-type strips on high resistivity n-type substrates. An ohmic contact (high doped n-type) is implanted on the back-plane (p-in-n diode structure). After inversion the junction (and the high electric field) migrates on the back-plane contact and a better charge collection is obtained by segmenting the n-type implant (n-in-n) [27].

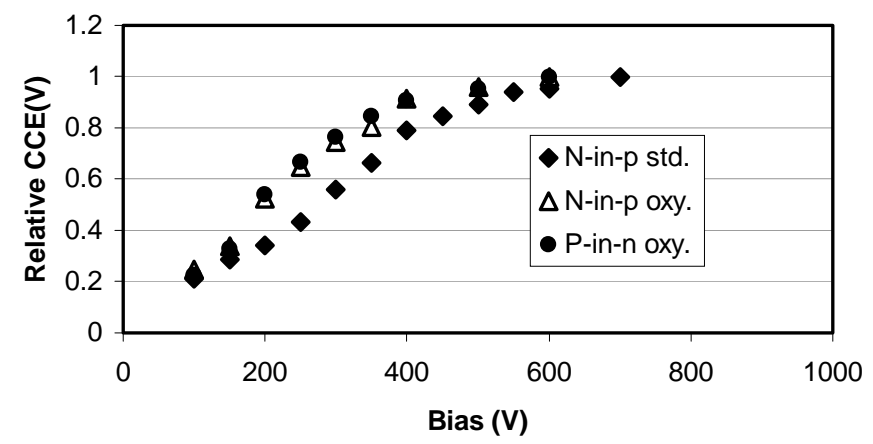

Figure 5. Charge collection of oxygenated and standard n-in-p detectors in diode configuration after $1.1 \times 10^{15} \mathrm{~cm}^{-2}$ (24GeV proton). The collected charge is normalised to the maximum charge collected at 700 volts. 
The n-side read out can be implemented on n-type or p-type substrates. The use of p-type substrates has the advantages of avoiding type inversion and providing the cheapest processing cost, due to the possibility of one side processing, compared to the double side processing required by n-type silicon substrates. In previous works, a substantial improvement of charge collection at low bias voltages was observed with standard p-type substrate after irradiation when compared to the p-side read-out [28].

A possible further improvement of the radiation hardness could be obtained with oxygen enriched p-type substrates. We investigated the charge collection properties of microstrip $n$-in-p detectors made with oxygenated p-type $\mathrm{Si}$ substrates, comparing their performances with p-in-n standard and oxygenated Si detectors [29]. P-type wafers have been oxygen enriched by CNM Barcelona to produce miniature micro-strip detectors for the first time with this substrate.

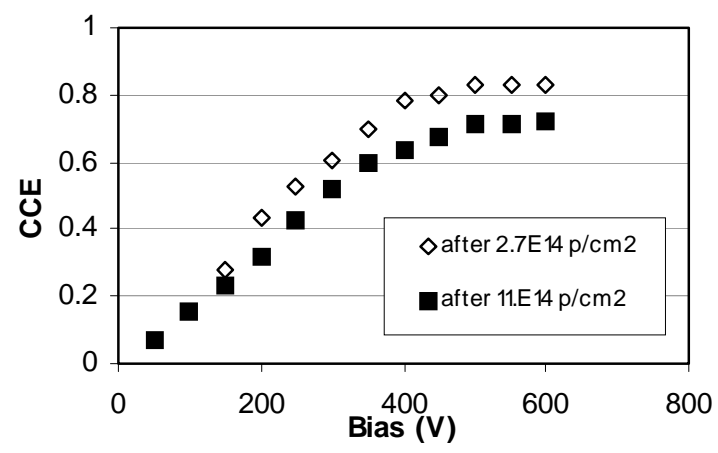

Figure 6. Charge collection efficiency, normalised to the pre-irradiation value, of oxygen enriched p-type substrate silicon detectors after 2.7 and $11.010^{14} \mathrm{p} \mathrm{cm}^{-2}$.

Two sets of oxygenated and standard detectors have been irradiated to 2.7 and $11.5 \times 10^{14} \mathrm{p} \mathrm{cm}^{-2}$ with $24 \mathrm{GeV}$ protons at the CERN-PS, at room temperature and unbiased. After irradiation they were kept at low temperature. Their charge collection properties have been measured in diode configuration, with all the $\mathrm{AC}$ coupled metal strips connected to a common rail and read out with a fast current amplifier $(<1 \mathrm{~ns}$ rise time). This technique is used to measure the maximum collected charge and the full depletion voltage, to provide a comparison of different substrates in "diode" configuration. The signal is though due to both electron and holes charge carriers and the advantage of n-side read out in segmented devices (mainly electron signal) is not evidenced. Figure 5 shows the $\operatorname{CCE}(\mathrm{V})$ curve, normalized to the value collected at $700 \mathrm{~V}$, for oxygenated and standard detectors after the highest radiation fluence for $\mathrm{p}$-in-n and n-in-p 
geometry. The relative charge collection at low biases is improved in the oxygen enriched detectors whatever geometry was used.

Figure 6 shows the charge collection efficiency, measured with fast electrons ( minimum ionising particles) from a ${ }^{106} \mathrm{Ru}$ source and normalized to the value before irradiation, of the oxygen enriched detectors for two irradiation fluences. These detectors show good charge collection properties also after heavy irradiation $\left(\sim 70 \%\right.$ of the charge is collected after $\left.1.1 \times 10^{15} \mathrm{p} \mathrm{cm}^{-2}\right)$. The oxygenated p-type detectors show a slightly higher charge collection at low bias voltages, after heavy irradiation, compared to standard p-type substrates, due to a lower value of $V_{\text {fd }}$. They both show a reduced dependence of $V_{f d}$ on the radiation fluence This may be due to the low resistivity starting silicon or to a reduced change in the effective space charge in this type of material. Further studies with initial higher resistivity p-type silicon are needed to demonstrate this option for lower cost high radiation tolerant detectors.

\section{New materials}

RD50 investigates the use of semiconductor, or semi-insulating, materials alternative to silicon with enhanced radiation hardness properties. The main focus is on silicon carbide, characterised by a large bandgap and with intermediate properties between $\mathrm{Si}$ and diamond. Many results have been obtained with bulk $(\sim 100 \mu \mathrm{m}$ thick) and epitaxial $(\sim 7-50 \mu \mathrm{m}$ thick) silicon carbide (SiC). The bulk semi-insulating single crystal $4 \mathrm{H}-\mathrm{SiC}, 550 \mu \mathrm{m}$ thick, was produced by Cree Research (USA) with high material resistivities $\left(>10^{11} \Omega \mathrm{cm}\right.$ ) and then thinned by lapping and polishing to $\sim 100 \mu \mathrm{m}$. The standard Schottky detectors produced with this material have been measured with $5.48 \mathrm{MeV}$ $\alpha$-particles with reverse bias voltages in the range 50-600 V. At the maximum reverse voltage the charge collection efficiency was found to be only about $60 \%$. This suggests the presence of defect levels that trap and recombine the charge before it can be collected. Better results in term of sensitivity to ionising radiation have been obtained with the epitaxial SiC. Schottky diodes made on a $20 \mu \mathrm{m}$ thick SiC epi-layer have been tested before and after irradiation (up to $10^{16} \mathrm{~cm}^{-2} 24 \mathrm{GeV} / \mathrm{c}$ protons, and $40 \mathrm{Mrad}$ with 8.2 electrons and $\gamma$-rays) to deliver $100 \%$ efficiency $[30,31]$.

Also gallium nitride $(\mathrm{GaN})$ epitaxial grown layers have been investigated by RD50 [32,33]. Preliminary results after irradiation of Schottky diodes made with this material have shown a CCE of $92 \%$ before irradiation and $77 \%$ after $5 \times 10^{14}$ neutrons $\mathrm{cm}^{-2}$, measure using a ${ }^{241} \mathrm{Am} \alpha$-particle source.

These results show that promising route to materials alternative to silicon are open and the progresses in crystal quality reflect in better detector performance. 


\section{Summary}

A wide range of activities are being performed by the RD50 to achieve the final goal of producing semiconductor detector able to survive the fluence of $10^{16}$ hadrons $\mathrm{cm}^{-2}$ and beyond. These activities involve the understanding the radiation damage at the microscopic level, the optimisation of the device geometry and the hunt for materials alternative to silicon. A short summary of these activities has been given here. A more detailed report can be found in [2].

\section{References}

[1] M. Hutinen, Nucl. Instr. and Meth. A 491 (2002) 194-215

[2] RD50 Status Report 2002/2003 CERN-LHCC-2003-058 and LHCC-RD002

[3] I. Pintilie, E. Fretwurst, G. Lindstroem and J. Stahl, Appl. Phys. Lett. 82, 2169 (2003).

[4] I. Pintilie, E. Fretwurst, G. Lindstroem and J. Stahl, Nucl. Inst. Meth. A 514 (2003) 18-24.

[5] J. Stahl et al., Physica B 340-342 (2003) 705-709.

[6] RD48, http://rd48.web.cern.ch/rd48/

[7] G. Lindström et al. (The RD48 Collaboration), Nucl. Instr. and Meth. A 466 (2001) 308.

[8] B.C. MacEvoy, A. Santocchia, G. Hall, Physica B 273-274 (1999) 1045

[9] B. Dezillie et al., IEEE Trans. Nucl. Sci. Vol. 47, No.6 (2000) 1892

[10] E. Fretwurst et al., Nucl. Inst. Meth. A 514 (2003) 1-8.

[11] G. Casse et al., Nucl. Instr. and Meth. A 438 (1999) 429-432.

[12] A. Barcz et al, Surface Science 203-204 (2003) 396.

[13] V. Savolainen et al., J. Crystal. Growth 243, 2 (2002) 243.

[14] J.L. Lindström, T. Hallberg, Phys. Rev. Lett. 72 (1994) 2729

[15] J. Härkönen et al., Nucl. Inst. and Meth. A514 (2003) 173-179.

[16] J. Stahl et al., Nucl. Inst. Meth. A 512 (2003) 111-116.

[17] G. Kramberger et al., Nucl. Inst. Meth. A 515 (2003) 665-670.

[18] J.L. Lindström et al., Physica B 308-310 (2001) 284

[19] Y.L. Lee et al., Phys. Rev. B 65 (2002) 085205

[20] J.L. Lindström, et.al., Materials Science Forum Vols 258-263(1997) pp. 367-372

[21] E. Tuominen et al., IEEE Trans. Nucl. Sci 50 (2003).

[22] Z. Li et al., Radiation Hardness of High resistivity CZ Si Detectors after Gamma, Neutron and Proton Radiations, $2^{\text {nd }}$ RD50 - Workshop 
on Radiation hard semiconductor devices for very high luminosity colliders, CERN (Geneva)18-20 May, 2003.

[23] G. Lindström et al., Improved Radiation Tolerance of Silicon Detectors for HEP Applications - Results from the CiS-HH Project 2nd RD50 Workshop on Radiation hard semiconductor devices for very high luminosity colliders, CERN (Geneva)18-20 May, 2003

[24] S I Parker, C J Kenney and J Segal 1997 Nucl. Instr. Meth. A 395328.

[25] S. Ronchin, M. Boscardin, G. F. Dalla Betta, P. Gregori, V. Guarnieri, C. Piemonte, N. Zorzi, Thin detectors fabrication at ITC-Irst , 3rd RD50 Workshop October 19-25, 2003.

[26] Z. Li on behalf of the US RD50: Activities of the US Chapter of CERN RD50 On the Development of Semi-3D Si Detectors, 2nd RD50 Workshop CERN, 18-20 May, 2003

[27] G. Casse et al., Nucl. Inst. and Meth. A511/1-2 (Sep. 2003) 112-117.

[28] G. Casse et al., IEEE Trans. Nucl. Sci., vol. 47, n. 3 (527-532), June 2000.

[29] G. Casse, First results with oxygenated n-in-p detectors after irradiation, $2^{\text {nd }}$ RD50 Workshop on Radiation hard semiconductor devices for very high luminosity colliders (CERN, May 18-20, 2003).

[30] F. Nava et al. Performance of Silicon Carbide Radiation Detectors, IEEE 2003 NSS MIC RTSD, Portland, Oregon, USA, October 19-25, 2004.

[31] M. Bruzzi et al., Recent results on particle detection with epitaxial SiC Schottky diodes, presented at the IEEE 2002 NSS-MIC Conference, Norfolk, Virginia, November 2002

[32] J.Vaitkus et al., Nucl. Inst. and Meth. A514 /1-3 (Nov. 2003) 141-145.

[33] J.V.Vaitkus et al., Polycrystalline Semiconductors VII. Volume 93-93 (2003) pp.301-306. 